Published in final edited form as:

J Struct Biol. 2016 August ; 195(2): 190-198. doi:10.1016/j.jsb.2016.06.001.

\title{
A new method for vitrifying samples for cryoEM
}

\author{
Ivan Razinkov\#, Venkat Dandey\#, Hui Wei, Zhening Zhang, David Melnekoff, William J. Rice, \\ Christoph Wigge, Clinton S. Potter, and Bridget Carragher \\ Simons Electron Microscopy Center, The National Resource for Automated Molecular Microscopy, \\ New York Structural Biology Center \\ \# These authors contributed equally to this work.
}

\section{Abstract}

\begin{abstract}
Almost every aspect of cryo electron microscopy (cryoEM) has been automated over the last few decades. One of the challenges that remains to be addressed is the robust and reliable preparation of vitrified specimens of suitable ice thickness. We present results from a new device for preparing vitrified samples. The successful use of the device is coupled to a new "self-blotting" grid that we have developed to provide a method for spreading a sample to a thin film without the use of externally applied filter paper. This new approach has the advantage of using small amounts of protein material, resulting in large areas of ice of a well defined thickness containing evenly distributed single particles. We believe that these methods will in the future result in a system for vitrifying grids that is completely automated.
\end{abstract}

\section{Keywords \\ CryoTEM; automation; self-blotting grids; nanowires}

\section{Introduction}

\begin{abstract}
Structure determination by cryo electron microscopy (cryoEM) has undergone a revolution over the past several years (Kühlbrandt, 2014) enabling near atomic resolution of a wide range of macromolecules. This revolution has been driven by a number of technological innovations in both hardware and software (Cheng, 2015). One of the challenges that remains in this field is the preparation of vitrified specimens (Adrian et al., 1984, Dubochet et al., 1988) of consistent and reliable quality. For cryoEM structure determination, protein samples must be suspended in a thin layer of vitrified buffer preferably supported by a holey substrate (typically carbon or gold foils) overlaid onto a metal mesh (e.g. gold, copper, molybdenum etc.) (Ermantraut et al., 1998) (Quispe et al., 2007) (Meyerson et al., 2014) (Russo and Passmore, 2016). The thin vitrified layer of sample is commonly produced by
\end{abstract}

\footnotetext{
Corresponding Author: Bridget Carragher, Simons Electron Microscopy Center, New York Structural Biology Center, 89 Convent Avenue, New York, NY 10027, (212) 939-0660; bcarr@ nysbc.org.

Supplementary Videos

Supplementary Video V1: Spotiton 1.0 going through a grid spotting cycle.

Supplementary Video V2: Video of ribosome sample as it is spotted onto a nanowire grid. The video ends when the sample is plunged into liquid ethane.
} 
pipetting a small volume of sample (typically $2-3 \mu \mathrm{l}$ ) onto the surface of a freshly plasma treated grid, and then removing most (>99.99\%) of the sample using filter paper to leave a thin wet layer (typically $\sim 100$ 's nm thick) across the surface. The wet grid is then rapidly plunged into a cryogen (e.g. liquid ethane, propane). The grid that results from this process is usually covered by a layer of vitreous ice of variable thickness; some holes are covered by ice that is too thick to allow imaging of the particles, some holes by ice that is so thin that the particles are excluded from the solution or damaged (Taylor and Glaeser, 2008), and some areas are completely dry. Ideally the ice should be just a little thicker than the largest diameter of the particles in the sample and the particles should be evenly distributed across the hole. There is no straightforward way to calculate the ice thickness with sufficient accuracy or find all potentially suitable areas using low magnification images. Thus searching for holes covered by ice of precisely the right thickness can be a time consuming and difficult task.

For relatively large $(>500 \mathrm{kD})$ and soluble proteins there is a higher tolerance for variations in ice thickness and the particles are often fairly well distributed across the holes, so optimizing sample preparation can be a reasonably straightforward process, perhaps requiring a few trials to get the sample concentration ideal and a few to vary conditions to optimize the extent of ice of the ideal thickness. However, for small proteins, and particularly membrane proteins, the process of optimizing vitrification of a sample can be a time consuming and frustrating endeavor and stands as a major bottleneck to rapid structure determination. The optimization process also consumes a large amount of protein, most of which is discarded on the used blotting paper, and requires many hours of expensive microscope time for screening.

Several years ago we developed a prototype of a new device for vitrifying samples for cryoEM (Jain et al., 2012). The device, called Spotiton, uses a piezo electric inkjet dispensing system integrated into a custom designed vitrification robot. We demonstrated that the system was capable of dispensing picoliter to nanoliter volumes of specimen onto precise areas of a sample grid and that the sample could be successfully vitrified. However, we acknowledged that spreading the sample into a suitably thin layer in the absence of mechanically applied blotting paper was an unsolved problem.

Here we report on our next generation of this device and on a new substrate that addresses the issue of spreading the specimen into a thin layer. We describe the device and the substrates in detail and demonstrate that we can vitrify a variety of samples to produce extensive areas of ice of uniform thickness and evenly distributed particles across holey substrates. The system has the advantage of using very small amounts of protein material, and provides ice of a well defined thickness in a well defined location thus avoiding the need for a time consuming search across the entire grid.

\section{Materials and Methods}

\section{Spotiton 1.0}

Overview-The basic steps required for robotic vitrification using Spotiton 1.0 are as follows (see also supplementary video V1). (i) A volume $(\sim 1 \mu \mathrm{L})$ of sample is aspirated into 
the piezo tip. (ii) The tip is washed in $\mathrm{ddH}_{2} \mathrm{O}$ to remove excess sample from the outer surface; this helps to avoid protein drying onto the surface and clogging the tip. (iii) The tip is wiped to remove any wash solution, which could interfere with the spot formation and precise targeting. (iv) The piezo head is positioned in front of a camera and spot formation is checked for size and accuracy. (v) The piezo head is positioned in front of the grid at a distance of $\sim 1 \mathrm{~mm}$ and records the drop location on the grid relative to the tip. (vi) Software is used to queue up a series of targets for spotting with a specified number of drops. (vii) The spots are dispensed based on the queued order. (viii) The grid is plunged into liquid ethane. (ix) The grid is transferred from the liquid ethane dewar into a grid box.

Spotiton 1.0 hardware design-During the development of Spotiton we have worked closely with Engineering Arts LLC, a precision engineering company based in Tempe, Arizona. Spotiton v1.0 is illustrated in Figure 1 and Supplementary Video S1. Extensive testing of the previous version (v0.75) of Spotiton (Jain et al., 2012) indicated a need for a new design to focus on improvements in: ease of use, motion control and repeatability, and piezo dispensing. The ease of use and reduced learning curve were addressed by introducing a single graphical user interface that controls all of the functions of the new robot. Improvements in motion were achieved by incorporating additional motion systems for the tweezers and improved actuators for the piezo dispensers. The steps required for vitrifying a grid are now almost all automated; these include: washing and drying the piezo dispensing tip, checking the sample spotting, aligning the grid and the tip location to ensure accurate targeting, queuing up multiple spots, plunging and transferring the grid from the ethane to a grid box. This high level of automation increases the repeatability of the experiments.

Spotiton 1.0 software integration-The various controls and outputs such as motion controllers, proximity sensors, actuators and video camera streams, were combined into a single software package. Previously, the user was required to keep track of two different video camera streams, one for checking of the proper droplet dispensing and a second for monitoring sample deposition onto the grids. The new version of the software automatically keeps track of the position of the tips and switches the camera feeds accordingly. Manual control of the video feed switching is still available for advanced users. During an experiment the software automatically creates and stores individual recordings of each sample deposition in a dated folder. The time required for sample deposition on the grid was reduced by implementing "click-to-move" and "click-to-spot" functionality into the software. During the start up procedure of the robot, each individual piezo dispensing tip is automatically calibrated to determine the relationship between its position on the video feed and the physical coordinates of the robot. Image recognition software is used to recognize the position of the piezo dispenser and match it with the XY-coordinates of the video feed. After calibration, the user is able to click anywhere on the live video and the tip moves to the new position. An option is implemented that allows users to deposit the sample by clicking on the video feed, effectively linking the motion of the robot with the activation of the dispensing units. The user also has the option of queuing up several targets on the grid for spotting the sample at different locations. 
Improved motion robotics-The range of motion was improved by the addition of $\mathrm{X}$ and Y-axis actuators to the tweezer/grid subassembly of the robot. This additional freedom of motion allows the user to precisely position the grid in front of the camera using the software user interface. The grid position is then updated automatically resulting in rapid and repeatable motion of the grid to and from the camera. To prevent the possibility of the grid and piezo dispenser subassemblies colliding with each other or with peripheral components, the software includes "soft limits" and "safe points". Soft limits constrain the movement of any axis through software checks on allowed positions, rather than physical limits. Safe points refer to an out of the way position for either subassembly, where no other moving components can collide with it. Previously, the tweezers with the grid had to be positioned directly over the liquid ethane bowl. With the added freedom of XY-movement of the tweezers, the user is able to place the grid anywhere in front of the camera without concern for the plunging accuracy. During normal operation of the robot the piezo dispensers are either in a vertical orientation for sample aspiration and drop checking, or in horizontal orientation for sample dispensing. The rotation axis was updated from a pneumatically driven actuator to a harmonic drive. The accurate control of the position and velocity of the harmonic drive improves the rotation of the piezo dispensers effectively preventing unwanted sample leaking during hard stops after rotation and ensuring correct positioning on every rotation.

Automatic grid transfer-To make Spotiton v1.0 more robust and easier to use, the traditionally manual process of moving the vitrified sample grids from liquid ethane to liquid nitrogen has been automated. Current state-of-art cryo plunging robots rely on a single axis motion of the tweezers in the Z-direction (up and down). In Spotiton 1.0 the tweezers are mounted to an independent 3-axis motion system. This freedom of movement allows free positioning of the tweezers and the grid in front of the camera and the piezo dispensers. The additional $\mathrm{X}$ and $\mathrm{Y}$-axis of motion allows rapid movement of the grid from the liquid ethane dewar to a designated grid box in liquid nitrogen. The time to complete the transfer from ethane to nitrogen using Spotiton is as at least as fast as can be achieved manually. The actuation of the opening of the tweezers is done using a pneumatically driven lever that moves one side of the tweezers, while holding the other side stationary. A section of the tweezers was thinned out to allow for elastic bending of the metal upon pneumatic actuation at 80 psi.

\section{"Self-blotting" TEM grids}

We have applied a method for growing nanowires on a copper surface (Zhang et al., 2013), originally developed for separating oil and water mixtures, to create a "self-blotting" grid. A simple chemical treatment can be used to grow nanowires (consisting of $\mathrm{Cu}(\mathrm{OH})_{2}$ ) of consistent length and diameter (see figure 2 ) on a copper surface. The nanowires are grown by floating Copper grids on top of a $20 \mu \mathrm{L}$ drop of a solution of $0.13 \mathrm{M}$ Ammonium Persulfate and $2.5 \mathrm{M} \mathrm{NaOH}$. We use a composite grid consisting of a sandwich of copper and palladium (see figure $2 \mathrm{~b}$ ) so that the nanowires are confined to the single top copper surface of the grid, leaving a smooth bottom palladium surface to which a holey carbon substrate can be adhered. The conditions for making the self-blotting grids used to vitrify samples shown in this paper were as follows. Copper-Palladium 150 mesh grids (Electron 
Microscopy Sciences, G150-CP) were cleaned by sonicating in acetone for 1 minute, followed by 1 minute sonication in ethanol, a wash in $\mathrm{ddH} 2 \mathrm{O}$ and drying in air. The grids were then floated copper side down on a $20 \mu \mathrm{l}$ drop of $0.13 \mathrm{M}$ Ammonium Persulfate and $2.5 \mathrm{M} \mathrm{NaOH}$ solution for 3-6 minutes, after which they were transferred to float on two sequential drops of ddH2O to stop the reaction. The grids were then dried on filter paper and examined under a light microscope to verify nanowire formation.

Holey carbon substrates were made in house using the method described in (Fukami and Adachi, 1965). Glass slides were cleaned with acetone, soaked in $0.03 \%$ tween 20 for 2 hours, and then rinsed in $\mathrm{ddH}_{2} \mathrm{O}$. Once dry, the slides were transferred to a chamber supporting humidity $10 \%$ points higher than measured ambient conditions. A continuous smooth thin layer of $0.25 \%$ Triafol in ethyl acetate was immediately applied to the slide, which was then allowed to dry inside the humidity chamber. The thin layer of plastic was floated off onto a water surface by slowly immersing the slide into water at an angle of about $40^{\circ}$. The previously prepared nanowire grids were then gently placed on top of the floating plastic with the copper (nanowire) side up. The plastic coated grids were lifted from the water using filter paper and allowed to dry. A thin layer of carbon was deposited onto the plastic coated nanowire girds using a Leica EM ACE600 coater with an in-situ crystal carbon thickness monitor, evacuated to a pressure of $3.510^{-5}$ torr. Extensive testing showed that a carbon layer of $10-30 \mathrm{~nm}$ is sufficient in strength and still supports thin ice across the holes. After carbon coating, the grids were baked at $65^{\circ} \mathrm{C}$ overnight which in our experience strengthens the carbon and helps to reduce contamination (Griffin et al., 2007). The plastic was then removed by quickly dipping the grid into acetone. The grid can then be stored for later use.

\section{Specimens}

Three specimens were used to demonstrate the capabilities of Spotiton and nanowire grids: 70S ribosomes (2MDa; New England Biolabs, P0763S, $13.3 \mu \mathrm{M}, 33.3 \mathrm{mg} / \mathrm{ml}$ ), hemagglutinin ( 200kDa, HA/Hong Kong/1/1968; My BioSource, Inc.,1mg/ml), Apoferritin (400kDa; Sigma Aldrich, A3660, 2.3mg/ml). Grids were treated using a Solarus plasma cleaner (see Table 1) just prior to sample preparation. Conditions used to make the grids are summarized in Table 1 and a video of the 70S ribosome spotting is provided in Supplementary Video 2.

TEM imaging and image processing-Data was acquired using Leginon MSI (Suloway et al., 2005) on a JEOL JEM3200FSC with an in column Omega energy filter operating at $300 \mathrm{KeV}$ and equipped with DE20 direct electron detector. The instrument was chosen based on its availability and only a modest set of images was acquired for each sample to provide a demonstration of the capabilities of this new vitrification method.

2D class averages were calculated for the $70 \mathrm{~S}$ ribosome dataset using the Appion image processing pipeline (Lander et al., 2009); CTF was estimated using Ctffind4 (Rohou and Grigorieff, 2015), a small set of particles were initial selected manually from a few micrographs and this small stack was used to calculate 2D class averages using the CL2D algorithm (Sorzano et al., 2010). These class averages were then used as templates to pick particles for the rest of the micrographs using FindEM (Roseman, 2004). The final stack 
contained 10,880 particles and these were used to calculate class averages using XMIPP ML2D (Scheres et al., 2005).

SEM Imaging-For room temperature SEM imaging, grids were loaded into a grid holder and inserted into an FEI Helios Nanolab 650 FIB/SEM. Secondary electron (SE) images were collected at various magnifications at $2 \mathrm{keV}$ high tension, $200 \mathrm{pA}$ current with a dwell time of $3 \mu$ s and an x-resolution of 1024 pixels across.

For cryo-imaging, grids were clipped at liquid nitrogen temperature into FEI Autogrid cartridges, with all manipulations performed inside a cryo-ultramicrotome (UC6, Leica Microsystems $\mathrm{GmbH}$, Hersteller, Germany). Cartridges were inserted into a grid-holding shuttle (described in (Rigort et al., 2010)), and transferred into the FIB/SEM using a cryotransfer system (PP3000T, Quorum Technologies, Laughton, Lewes, East Sussex, Great Britain). The stage was held at a constant temperature of $-180{ }^{\circ} \mathrm{C}$ during imaging. Imaging was performed at $2 \mathrm{keV}$ high tension, $200 \mathrm{pA}$ current. To prevent charging artifacts, SE images were collected with a dwell time of $0.1 \mu$ s and integrated $32 \times$ over the specimen with an $\mathrm{x}$-resolution of 512 pixels across. At the end of the experiment, column valves were closed and the stage was heated to $-60{ }^{\circ} \mathrm{C}$ in order to sublime all of the ice from the sample. After approximately 15 minutes, all ice was removed and the bare nanowires were fully visible.

\section{Results and Discussion}

We have demonstrated that we can vitrify a variety of samples (70S ribosomes, HA, Apoferritin) using Spotiton to apply small volumes ( $2.5 \mathrm{~nL}-16 \mathrm{~nL})$ of sample to nanowire grids adhered to holey carbon substrates. The nanowires effectively act as blotting paper to whisk the sample from the surface as soon as the liquid comes into contact with the nanowires (see Figure 3 and Supplementary Video V2) leaving behind a thin wet film that is then plunged into liquid ethane for rapid vitrification. Below we provide some details of various aspects of this process.

The nanowires can be grown on a variety of different sized copper mesh grids; we have tested 400, 300, 200, 150, 100 and 75 mesh. Here we have used 150 mesh grids because the squares are of a size that is straightforward to target and vitrify using our current Spotiton system. The dimension of a grid square at this mesh size is $\sim(125 \mu \mathrm{m})^{2}$ and there are several thousand holes covering a single square for the lacey carbon substrates used in these experiments.

The density and the number of nanowires can be controlled by the length of time the copper is exposed to the chemical treatment. Figure 4 shows images acquired using a scanning electron microscope (SEM) that illustrate the range of densities and lengths of nanowires that can be achieved by varying chemical exposure times. In general, the length of the nanowires appears to be in the range of 3-5um, for both low ( $10 \%$ area coverage), medium ( $75 \%$ coverage) and high ( $100 \%$ coverage) density growth. The nanowires only grow on the copper surface leaving the palladium surface bare and smooth; this is important on order to firmly adhere a smooth and strong carbon substrate to the grid. 
While we can generally control the density of the nanowires for a given batch of commercial copper grids, the nanowire growth sometimes varies from batch to batch, likely indicating impurities in the copper. We will address this in the future by manufacturing our own copper sandwich grids by evaporating copper onto pure gold grids. We have shown that nanowires will grow on a layer of copper of $\sim 80 \mathrm{~nm}$ thick and that the gold surface remains free of nanowires and ready for substrate adherence. This approach will also have the advantage that we can then provide gold substrate on a gold mesh that has been shown to be effective in reducing beam induced movement (Meyerson et al., 2014, Russo and Passmore, 2016) although it remains to be seen what effect the metal sandwich construction will have on beam induced movement.

The wicking properties of the nanowire grids are dependent on several factors of which the most critical are the density and length of the nanowires and the length of time and method used to plasma clean the grids prior to sample preparation. Figure 3 provides three individual frames from a video showing a grid as a 20pL spot is applied (figure 3a), 200 msecs after spotting (figure $3 \mathrm{~b}$ ) and 400 msecs after spotting, at which point the grid would be plunged into ethane.

The nanowires are very efficient at removing sample from the grid substrate. Although, we have the option to deliver sample volumes as low as $\sim 1 \mathrm{~nL}$ we use $20-40 \mathrm{~nL}$ in practice in order to ensure that a wet film remains on the grid at the time of plunging. This aspect is limited by the current version of Spotiton that requires on the order of one second to withdraw the tip and then deliver the grid into the liquid ethane; this relatively long time lapse means that smaller volume spots are completely wicked away to leave a dry surface by the time the grid enters the cryogen. In the future, we plan to address this issue by speeding up the timing between spotting and plunging, our goal is to reduce the time between spotting and plunge to $\sim 50 \mathrm{msecs}$. This will be achieved by a redesign of the geometry of the tweezers and the plunge axis and by spotting to the grid after it is already moving on a vertical path into the liquid ethane. Our goal would be to time the spots, and the vertical axis velocity so that a series of spots would be applied across the grid as it moves past the tip. In this way, we can apply spots to several grid squares and, as the travel time will ensure a range of wicking times, this should help in creating a range of grid squares around a desired ice thickness. Improving the spot to plunge time will also reduce the effects of evaporation of the thin wet film. Currently, we estimate that under the conditions we are using $(\sim 80 \% \mathrm{RH}$, $25^{\circ} \mathrm{C}$ ), a layer of $\sim 100 \AA$ thick evaporates form the surface of the thin layer, which itself is $\sim 1000 \AA$ thick as estimated from images obtained using the cryoSEM. This represents a $10 \%$ loss in volume with possible negative consequences for the stability of sensitive proteins. Speeding up the drop time will virtually eliminate any evaporation and in the next version of the instrument we will install a system to carry air from HEPA filter air circulators to control the local environment around the grid $\left(20-95 \% \mathrm{RH}, 4^{\circ}-25^{\circ} \mathrm{C}\right)$.

In the videos of the spotting process it appears that the sample volume is soaked up preferentially along the length of the nanowires rather than flowing into neighboring squares. In examining the resulting grids in the TEM we can confirm that areas of vitrified ice are almost invariably confined to the square that is targeted; the only exception is an occasional off target spotting that lands on the wires between two squares and will then 
usually flow into both of the neighboring squares, this is the case with the spotting shown in Supplementary Video V1 of the ribosome experiment. In order to understand how the liquid is taken up by the nanowires we preserved one of the spotted vitrified grids after examining it in the TEM and transferred it under cryo conditions to an SEM. SEM images acquired under cryo conditions are shown Figure $2 \mathrm{c}$-e. The two squares in the center are covered by a thin layer of ice while the surrounding grid squares are dry; this was also observed during the prior TEM imaging session. The nanowire covered grid bars are coated in a thick layer of ice that appears to fill up the entire volume provided by the nanowires. Figure $2 \mathrm{f}$ shows an area of the same gird after removal of the ice by heating. The ice layer on the nanowires extends from the square that was spotted all the way out along the nanowires to the edge of the grid in all directions; figure $2 \mathrm{e}$ shows an area near the edge of the grid, 7 grid squares away from the center spot. The observation that the nanowires are soaked and appear to be filled to capacity is consistent with our observation that the wicking speed of the grid appears to decrease fairly linearly when applying $10(4 \mathrm{~nL}), 20(8 \mathrm{~nL})$, or $30(12 \mathrm{~nL})$ drop volumes but falls off when going up to $40(16 \mathrm{~nL})$ drops. See Table 2 . These data are also consistent to an order of magnitude with our calculation of the volume of liquid (10nL) that could be absorbed by a layer of nanowires $5 \mu \mathrm{m}$ in length covering the entire surface of the 150 mesh grid. The volume of liquid spread to a thin film across the individual grid square is $\sim 2 \mathrm{pL}$.

The present dispenser nozzle on Spotiton aspirates $\sim 1 \mathrm{uL}$ of sample, but the amount of sample that is required to spot one square of the grid is $\sim 10 \mathrm{~nL}$. Thus the $1 \mathrm{uL}$ starting volume is sufficient to make $\sim 100$ grids! In practice right now we use some of the volume to test our an area of the grid in a peripheral region to get the volume and timing right for final spotting to the middle of the grid. Typically this requires 2-4 attempts but even so there is enough sample for many grids. Once an on-the-fly spotting system is developed this testing phase should no longer be necessary. We will also explore a variety of ways of reducing the sample dead volume so that smaller starting volumes can be used.

We show images from 3 samples in this paper to demonstrate the capabilities of this new method of vitrification: 70S ribosomes (figure 5), hemagglutinin (HA) (figure 6) and Apoferritin (figure 7). All of these experiments have been repeated several times and we have shown that we can achieve consistent and predictable results. Overall the grids produced using this new approach result in a layer of good quality vitrified ice of consistent thickness that is evenly spread over the entire grid square. Individual particles within the holes are also fairly evenly and well distributed. As a result, within a single square of a nanowire grid we have as many as several 1000 individual holes covered in vitreous ice of appropriate thickness and particle distribution. Thus an entire dataset could readily be obtained form a single grid square on a single nanowire grid prepared using Spotiton. This improves the efficiency of the data collection process as all data is acquired from a known position on the grid eliminating the need to search the grid for squares that provide holes covered by a layer of ice of suitable thickness.

There are many more avenues to explore with this new technology and many possibilities for further progress. As described above we have already begun to create copper coated gold grids that can then be adhered to a gold holey substrate; we will need to investigate whether 
the stability of these copper/gold sandwich grids is comparable to the pure gold grids. We will also make changes to the basic engineering of Spotiton to speed up the spot to plunge time. Our goal will be to reduce the interval between sample spotting and vitrification to 10 's of milliseconds. This will give us much more control over the overall system and enable more exacting settings for ice thickness. It will also be straightforward to enable spotting multiple samples very rapidly and, combined with rapid plunging, this will be used to explore if the system can be used to mix samples on the grid in order to explore time resolved events. We believe that this new approach combined with the self-blotting nanowire grids opens up the future possibility of a fully automated method for preparing vitreous ice grids.

\section{Supplementary Material}

Refer to Web version on PubMed Central for supplementary material.

\section{Acknowledgements}

We thank Peter Kahn and Al Roper at Engineering Arts for building our Spotiton devices and for much useful discussion and input. Thanks to Ed Eng, Carl Negro, Ashleigh Raczkowski, Yong Zi Tan and other members of the Simons Electron Microscopy Center for help at various stages. We would like to acknowledge primary financial support from the National Institutes of Health (NIH) National Institute of General Medical Sciences (GM103310 to A.C., C.S.P., B.C.); and the Simons Foundation (349247 to C.S.P., B.C.)

\section{References}

ADRIAN M, DUBOCHET J, LEPAULT J, MCDOWALL AW. Cryo-electron microscopy of viruses. Nature. 1984; 308:32-6. [PubMed: 6322001]

CHENG Y. Single-particle Cryo-EM at crystallographic resolution. Cell. 2015; 161:450-457. [PubMed: 25910205]

DUBOCHET J, ADRIAN M, CHANG J, HOMO J, LEPAULT J, MCDOWALL A, SCHULTZ P. Cryo-electron microscopy of vitrified specimens. Q Rev Biophys. 1988; 21:129-228. [PubMed: 3043536]

ERMANTRAUT E, WOHLFART K, TICHELAAR W. Perforated support foils with pre-defined hole size, shape and arrangement. Ultramicroscopy. 1998; 74:75-81.

FUKAMI A, ADACHI K. A new method of preparation of a self-perforated micro plastic grid and its application. J Electron Microsc (Tokyo). 1965; 14:112-8. [PubMed: 5852585]

GRIFFIN B, JOY D, PROBST C, SUVOROVA A, WALCK S, GAUVIN R. Texture and Stability and the Effect of the Sample on Thin Metal Film Coatings for High Resolution and Low Voltage SEM. Microscopy and Microanalysis. 2007; 13:1074-1075.

JAIN T, SHEEHAN P, CRUM J, CARRAGHER B, POTTER CS. Spotiton: a prototype for an integrated inkjet dispense and vitrification system for cryo-TEM. J Struct Biol. 2012; 179:68-75. [PubMed: 22569522]

KÜHLBRANDT W. The resolution revolution. Science. 2014; 343:1443-1444. [PubMed: 24675944]

LANDER GC, STAGG SM, VOSS NR, CHENG A, FELLMANN D, PULOKAS J, YOSHIOKA C, IRVING C, MULDER A, LAU PW, LYUMKIS D, POTTER CS, CARRAGHER B. Appion: an integrated, database-driven pipeline to facilitate EM image processing. J Struct Biol. 2009; 166:95102. [PubMed: 19263523]

MEYERSON JR, RAO P, KUMAR J, CHITTORI S, BANERJEE S, PIERSON J, MAYER ML, SUBRAMANIAM S. Self-assembled monolayers improve protein distribution on holey carbon cryo-EM supports. Sci Rep. 2014; 4:7084. [PubMed: 25403871] 
QUISPE J, DAMIANO J, MICK SE, NACKASHI DP, FELLMANN D, AJERO TG, CARRAGHER B, POTTER CS. An improved holey carbon film for cryo-electron microscopy. Microsc Microanal. 2007; 13:365-71. [PubMed: 17900388]

RIGORT A, BAUERLEIN FJ, LEIS A, GRUSKA M, HOFFMANN C, LAUGKS T, BOHM U, EIBAUER M, GNAEGI H, BAUMEISTER W, PLITZKO JM. Micromachining tools and correlative approaches for cellular cryo-electron tomography. J Struct Biol. 2010; 172:169-79. [PubMed: 20178848]

ROHOU A, GRIGORIEFF N. CTFFIND4: Fast and accurate defocus estimation from electron micrographs. J Struct Biol. 2015; 192:216-21. [PubMed: 26278980]

ROSEMAN AM. FindEM--a fast, efficient program for automatic selection of particles from electron micrographs. J Struct Biol. 2004; 145:91-9. [PubMed: 15065677]

RUSSO CJ, PASSMORE LA. Ultrastable gold substrates: Properties of a support for high-resolution electron cryomicroscopy of biological specimens. J Struct Biol. 2016; 193:33-44. [PubMed: 26592474]

SCHERES SH, VALLE M, NUNEZ R, SORZANO CO, MARABINI R, HERMAN GT, CARAZO JM. Maximum-likelihood multi-reference refinement for electron microscopy images. J Mol Biol. 2005; 348:139-49. [PubMed: 15808859]

SORZANO CO, BILBAO-CASTRO JR, SHKOLNISKY Y, ALCORLO M, MELERO R, CAFFARENA-FERNANDEZ G, LI M, XU G, MARABINI R, CARAZO JM. A clustering approach to multireference alignment of single-particle projections in electron microscopy. J Struct Biol. 2010; 171:197-206. [PubMed: 20362059]

SULOWAY C, PULOKAS J, FELLMANN D, CHENG A, GUERRA F, QUISPE J, STAGG S, POTTER CS, CARRAGHER B. Automated molecular microscopy: the new Leginon system. J Struct Biol. 2005; 151:41-60. [PubMed: 15890530]

TAYLOR KA, GLAESER RM. Retrospective on the early development of cryoelectron microscopy of macromolecules and a prospective on opportunities for the future. J Struct Biol. 2008; 163:214-23. [PubMed: 18606231]

ZHANG F, ZHANG WB, SHI Z, WANG D, JIN J, JIANG L. Nanowire-Haired Inorganic Membranes with Superhydrophilicity and Underwater Ultralow Adhesive Superoleophobicity for HighEfficiency Oil/Water Separation. Advanced Materials. 2013; 25:4192-4198. [PubMed: 23788392] 

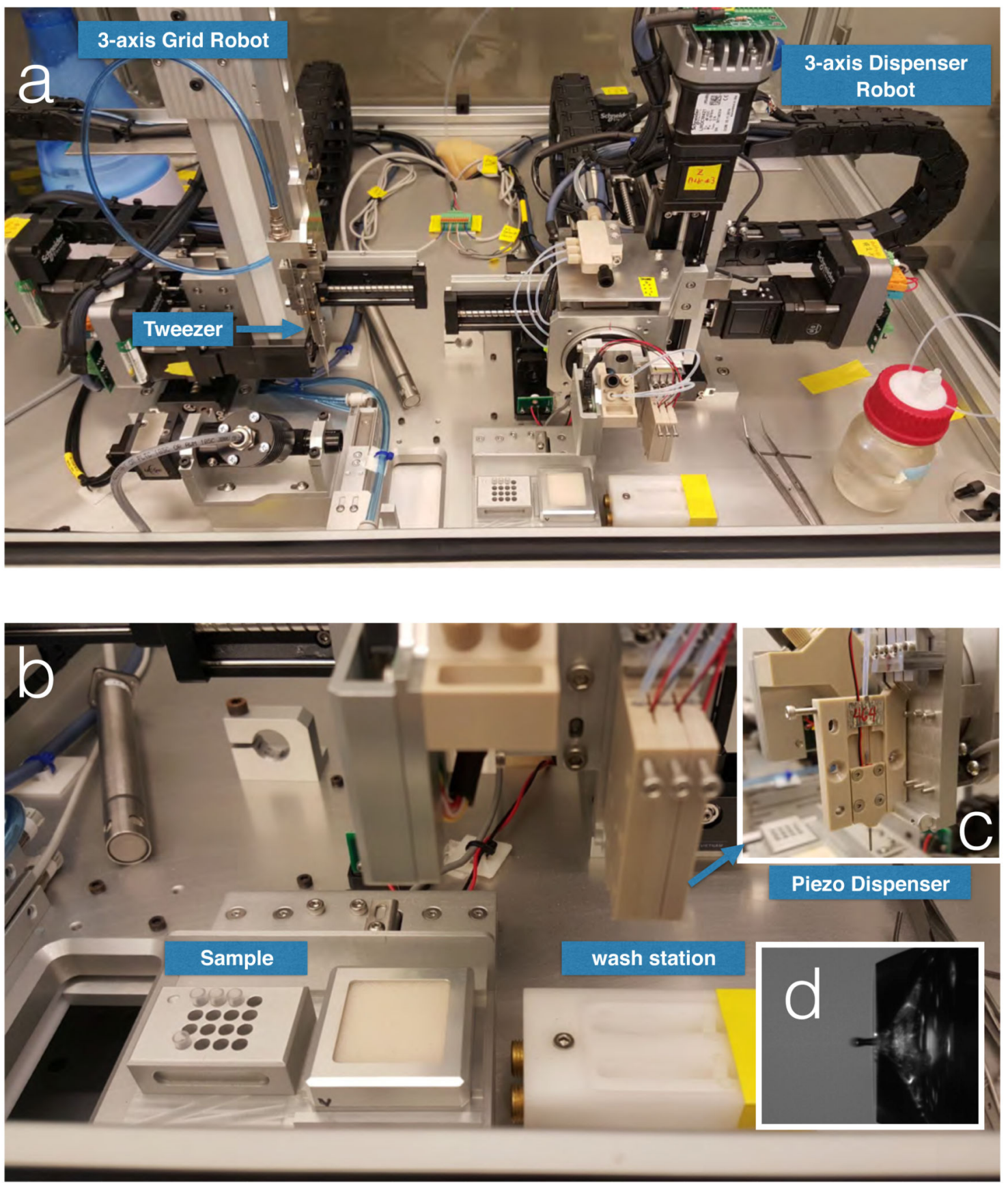

Figure 1.

Spotiton 1.0 device.

(a) Picture of the Spotiton 1.0 robot; a 3 axis robot grid arm can move the tweezers in three directions to precisely locate the grid in front of the tip; a independent 3-axis dispenser robot arm can move the tip to exact specified locations on the grid. (b) Close up views of the piezo tip dispenser and (c) washing and drying station. (d) View of drop in flight from piezo top. Minimum drop volume is $30 \mathrm{pL}$. 

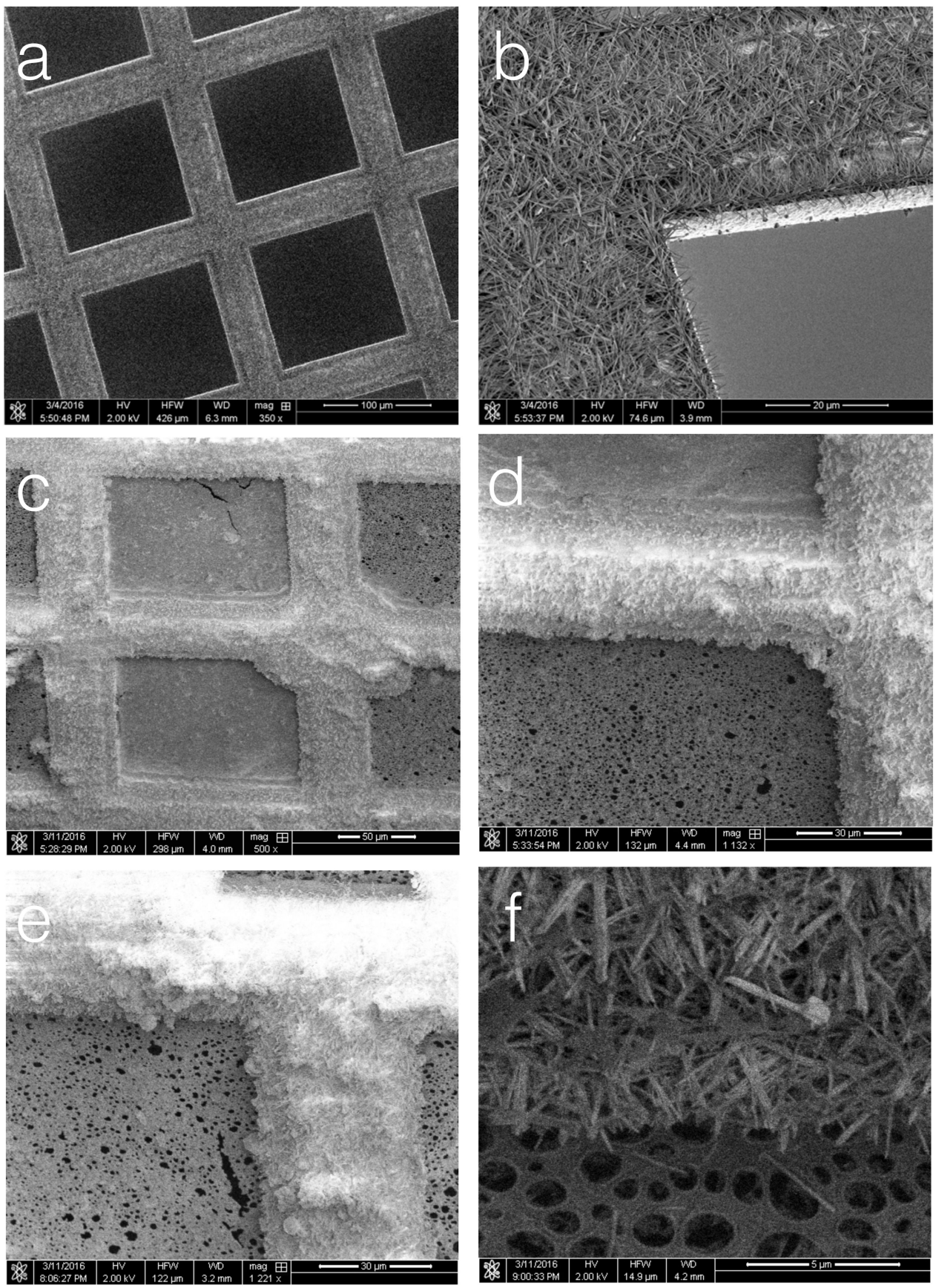

Figure 2.

Nanowire grids.

$(a, b)$ SEM images of a nanowire grid (after 6.5 minutes of chemical treatment). (c) After examination in a TEM, a vitrified nanowire grid was transferred to the cryo-SEM. Ice can be seen covering two squares of the grid (as confirmed by the TEM images and the video of the spotting). (d) Ice is observed to thickly coat the nanowires that appear to be saturated. (e) the saturation of the nanowires continues along the grid bars all the way out to the edge of the grid. (f) The nanowires after being cleared of ice by heating. 

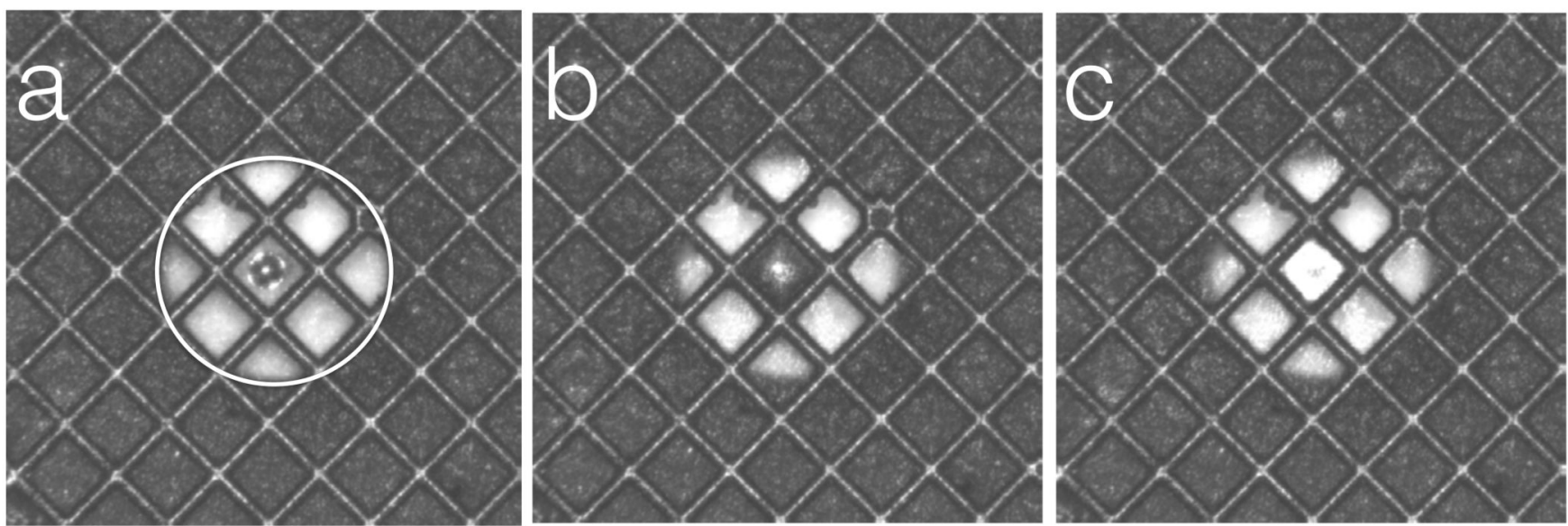

Figure 3.

Individual frames from a Spotiton video.

(a) Frame acquired just as the spot hits the surface of the grid. (b) 200msecs later the spot has contacted the nanowires and the liquid is being absorbed along the grid bars. (c) 200 msecs later the spot has been spread to a thin film. Note that the bright circular region, outlined by a circle in (a) is an image of the piezo tip. 

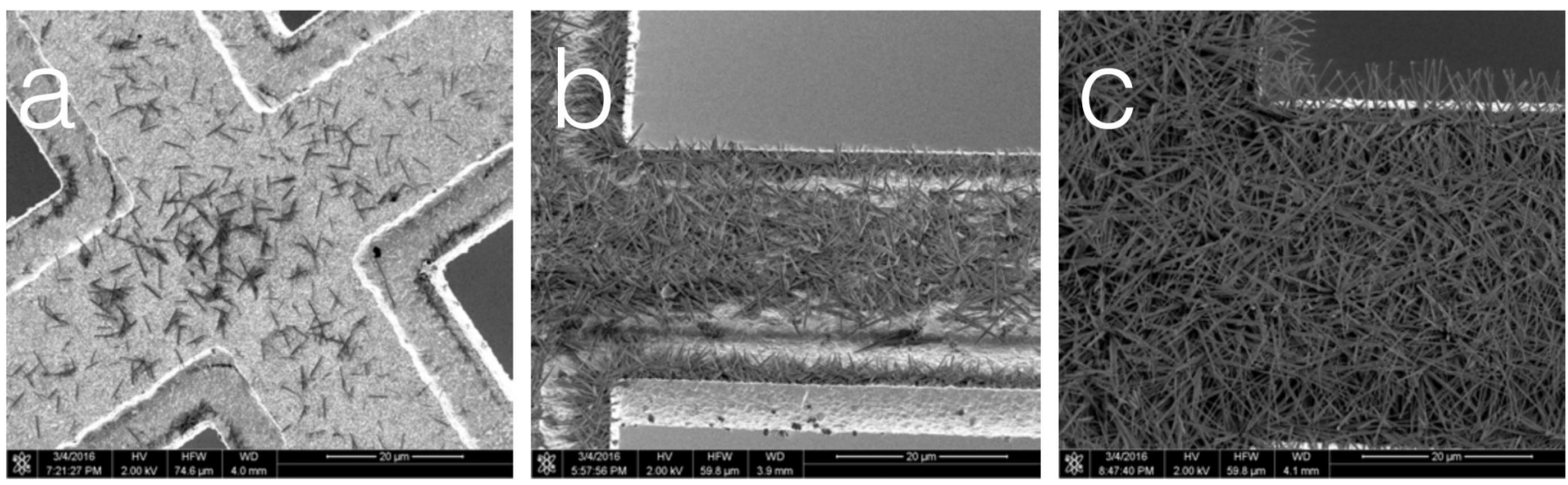

Figure 4.

Nanowire grids with varying lengths of time of chemical treatment in Ammonium Persulfate solution.

(a) 4 minutes, (b) 6 minutes, (c) 10 minutes. 

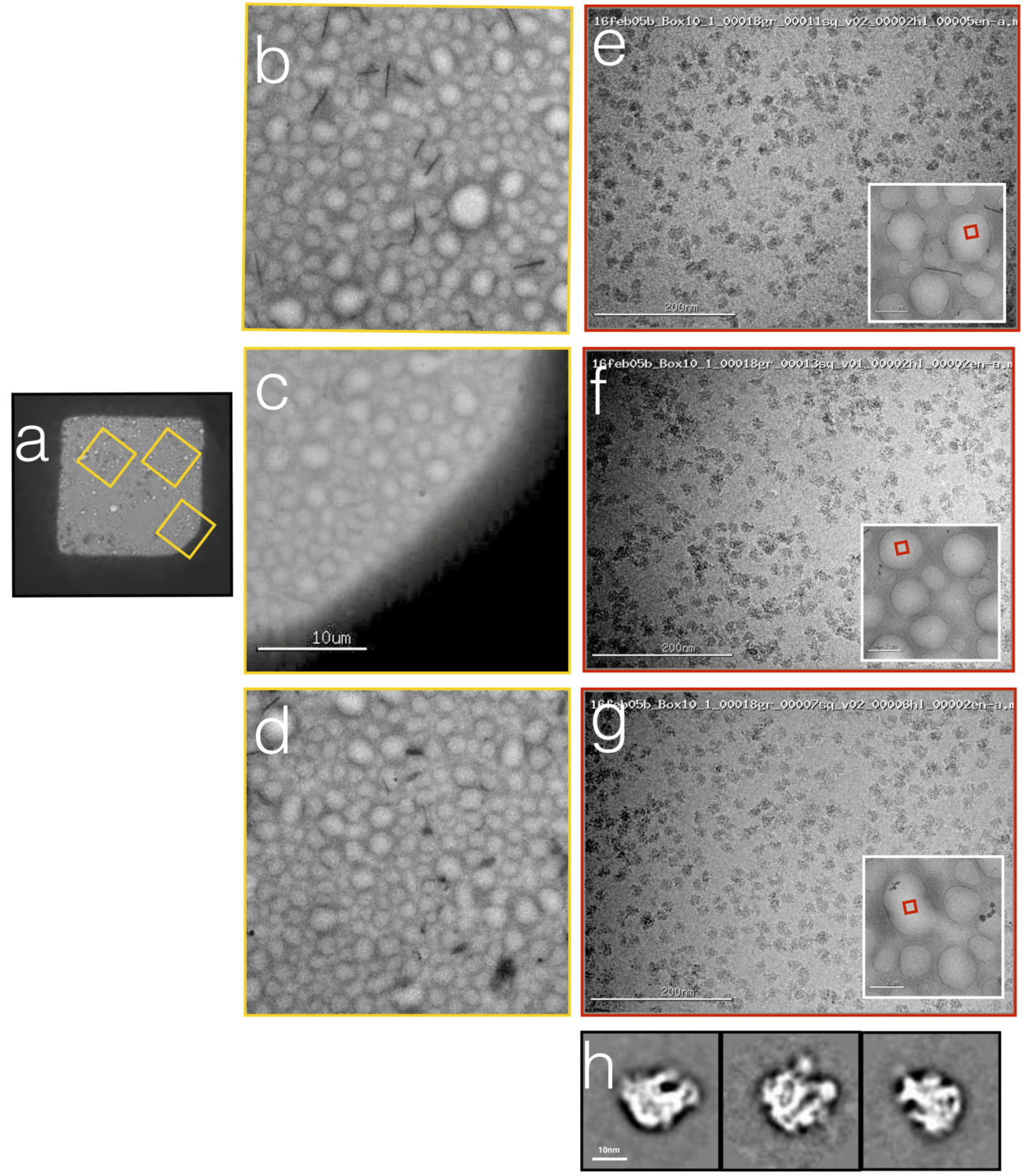

Figure 5.

Ribosomes vitrified onto nanowire grids using Spotiton.

(a) An image of the grid square into which the sample was spotted. (b,c,d) Larger scale views of the areas of the square indicated by the yellow boxes in (a). (e,f,g) High magnification images acquired from these regions of the grid square show a uniform ice thickness and fairly uniform distribution of particles across the holes. The red box in the inset indicates the exact hole that was imaged. (h) Representative class averages calculated form a small set of selected particles. 

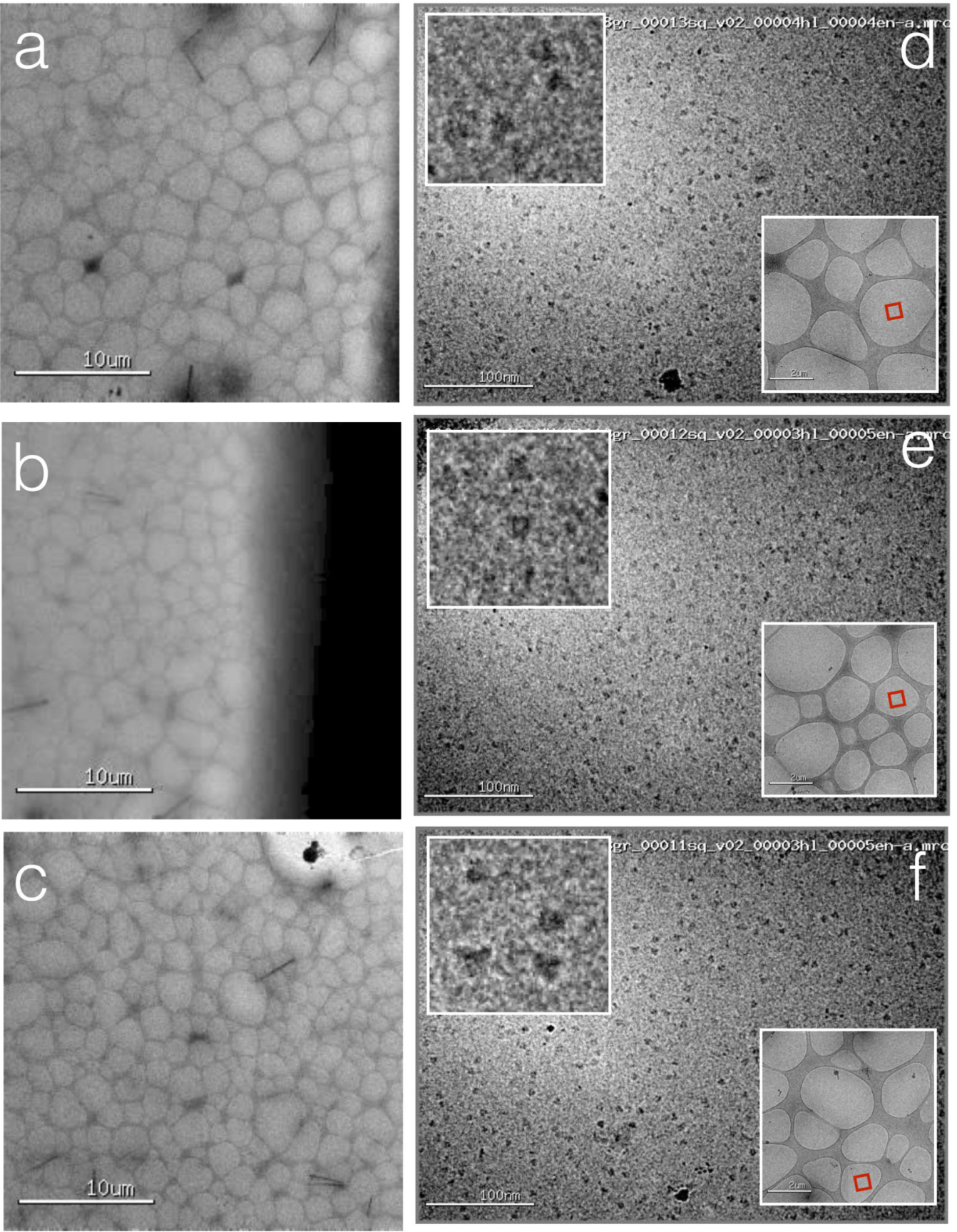

Figure 6.

Hemaglutinnin (HA) vitrified onto nanowire grids using Spotiton.

$(a, b, c)$ Several regions across the grid square that was spotted. (d,e.f) High magnification images acquired from these regions of the grid square show a uniform ice thickness and fairly uniform distribution of particles across the holes. The red box in the lower right inset indicates the exact hole that was imaged, the upper left inset shows a higher magnification image of a selected region. 

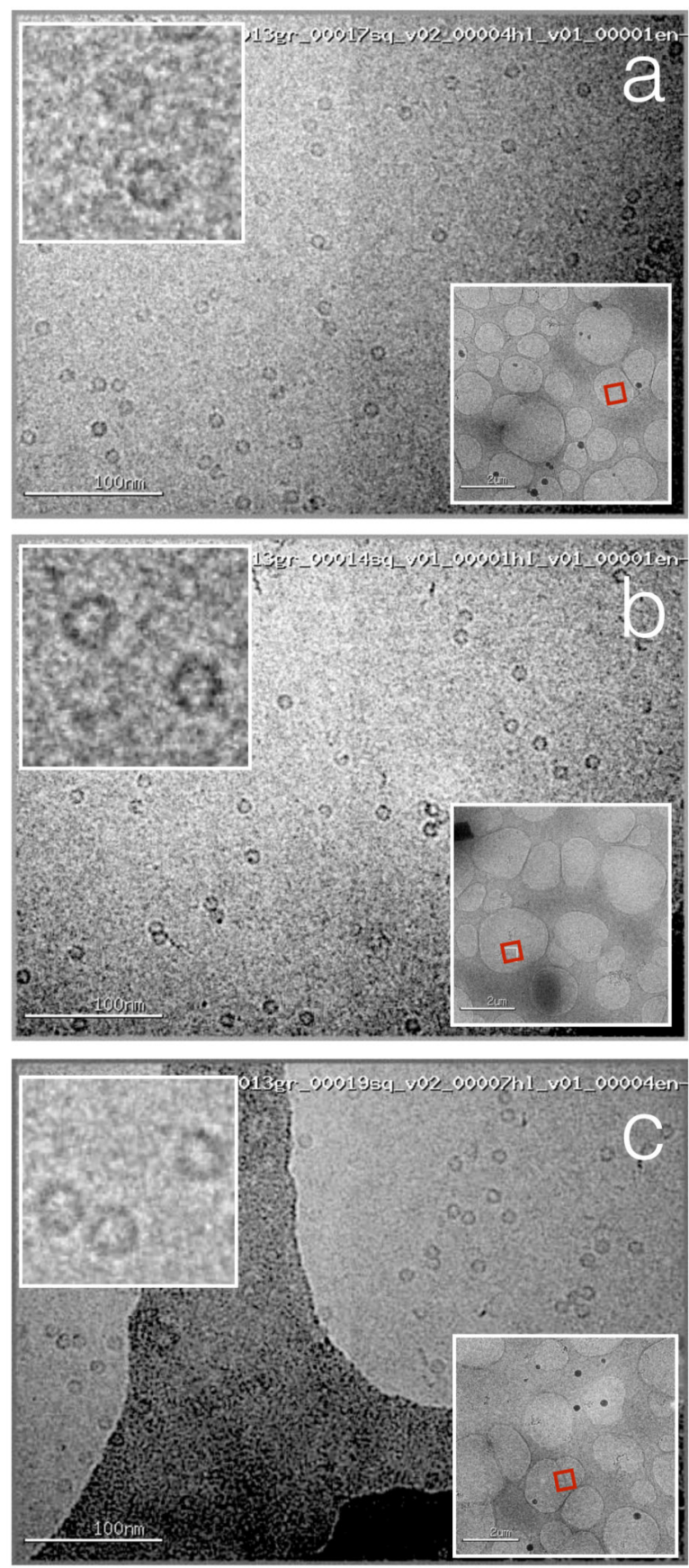

Figure 7.

Apoferritin vitrified onto nanowire grids using Spotiton.

$(a, b, c)$ High magnification images acquired from regions across the grid square show a uniform ice thickness and fairly uniform distribution of particles across the holes. The red box in the lower right inset indicates the exact hole that was imaged, the upper left inset shows a higher magnification image of a selected region. 


\section{Table 1}

Spotiton experimental parameters

\begin{tabular}{|l|l|l|l|l|l|}
\hline Sample & Concentration & $\begin{array}{l}\text { Plasma } \\
\text { treatment }\end{array}$ & $\begin{array}{l}\text { Sample } \\
\text { volume } \\
\text { spotted }\end{array}$ & $\begin{array}{l}\text { Relative } \\
\text { humidity; } \\
\text { temperature }\end{array}$ & $\begin{array}{l}\text { \# grid } \\
\text { squares } \\
\text { spotted }\end{array}$ \\
\hline $\begin{array}{l}70 \mathrm{~S} \\
\text { Ribosome }\end{array}$ & $0.33 \mathrm{mg} / \mathrm{ml}$ & $\begin{array}{l}20 \mathrm{secs} \\
\left(\mathrm{Ar}, \mathrm{H}_{2}\right)\end{array}$ & $40 \times 400 \mathrm{pL}$ & $\begin{array}{l}89 \% ; \\
22^{\circ} \mathrm{C}\end{array}$ & 2 \\
\hline Hemagglutinin & $0.1 \mathrm{mg} / \mathrm{ml}$ & $\begin{array}{l}15 \mathrm{secs} \\
\left(\mathrm{Ar}, \mathrm{H}_{2}\right)\end{array}$ & $35 \times 400 \mathrm{pL}$ & $\begin{array}{l}84 \% ; \\
23^{\circ} \mathrm{C}\end{array}$ & 1 \\
\hline Apoferritin & $0.2 \mathrm{mg} / \mathrm{ml}$ & $\begin{array}{l}15 \mathrm{secs} ; \\
\left(\mathrm{Ar}, \mathrm{H}_{2}\right)\end{array}$ & $40 \times 400 \mathrm{pL}$ & $\begin{array}{l}92 \% ; \\
23^{\circ} \mathrm{C}\end{array}$ & 3 \\
\hline
\end{tabular}




\section{Table 2}

Timing interval between spotting and observation that the substrate appears dry for nanowire grids spotted with a variety of sample volumes after varying intervals of dwell time inside an environment set at $80 \%$ humidity.

\begin{tabular}{|c|c|c|c|c|}
\hline $\begin{array}{l}\text { Grid dwell } \\
\text { time in } 80 \% \\
\text { humidity } \\
\text { chamber } \\
\text { before } \\
\text { spotting } \\
\text { (minutes) }\end{array}$ & $\begin{array}{l}10 \text { drops } \\
(4 \mathrm{~nL}) ; \\
\text { time to wick } \\
\text { dry }(\mathrm{s}) ; \text { rate of } \\
\text { volume } \\
\text { removal }(\mathrm{nL} / \mathrm{s})\end{array}$ & $\begin{array}{l}20 \text { drops } \\
(8 \mathrm{~nL}) ; \\
\text { time to wick } \\
\text { dry }(\mathrm{nL} / \mathrm{s}) ; \text { rate } \\
\text { of volume } \\
\text { removal }(\mathrm{nL} / \mathrm{s})\end{array}$ & $\begin{array}{l}30 \text { drops } \\
(12 n L) ; \\
\text { time to wick } \\
\text { dry }(\mathrm{nL} / \mathrm{s}) ; \text { rate } \\
\text { of volume } \\
\text { removal }(\mathrm{nL} / \mathrm{s})\end{array}$ & $\begin{array}{l}40 \text { drops } \\
(16 n L) ; \\
\text { time to wick } \\
\text { dry }(\mathrm{nL} / \mathrm{s}) ; \text { rate } \\
\text { of volume } \\
\text { removal }(\mathrm{nL} / \mathrm{s})\end{array}$ \\
\hline 0 & $0.20 \mathrm{~s} ; 20 \mathrm{~nL} / \mathrm{s}$ & $0.32 \mathrm{~s} ; 25 \mathrm{~nL} / \mathrm{s}$ & $0.44 \mathrm{~s} ; 27 \mathrm{~nL} / \mathrm{s}$ & $1.24 \mathrm{~s} ; 13 \mathrm{~nL} / \mathrm{s}$ \\
\hline 1 & $0.24 \mathrm{~s} ; 17 \mathrm{~nL} / \mathrm{s}$ & $0.40 \mathrm{~s} ; 20 \mathrm{~nL} / \mathrm{s}$ & $0.48 \mathrm{~s} ; 25 \mathrm{~nL} / \mathrm{s}$ & $1.0 \mathrm{~s} ; 16 \mathrm{~nL} / \mathrm{s}$ \\
\hline 2 & $0.32 ; 12 \mathrm{~nL} / \mathrm{s}$ & $0.44 \mathrm{~s} ; 18 \mathrm{~nL} / \mathrm{s}$ & $0.60 \mathrm{~s} ; 20 \mathrm{~nL} / \mathrm{s}$ & $0.96 \mathrm{~s} ; 17 \mathrm{~nL} / \mathrm{s}$ \\
\hline
\end{tabular}

